\title{
Genome Sequence of Colistin-Resistant Bacteremic Shewanella algae Carrying the Beta-Lactamase Gene bla $a_{\mathrm{OXA}-55}$
}

\author{
Ying-Ju Chen, ${ }_{1}^{1}$ Kwong-Chung Tung, ${ }^{2}$ Yu-Kai Hong, ${ }^{3}$ Shi-Yu Chen, ${ }^{3}$ Yao-Ting Huang $\mathbb{D}^{3},{ }^{3}$ \\ and Po-Yu Liu $\mathbb{i D}^{4,5,6}$ \\ ${ }^{1}$ Department of Food and Nutrition, Providence University, Taichung 43301, Taiwan \\ ${ }^{2}$ Department of Veterinary Medicine, National Chung Hsing University, Taichung 40227, Taiwan \\ ${ }^{3}$ Department of Computer Science and Information Engineering, National Chung Cheng University, Chiayi 62102, Taiwan \\ ${ }^{4}$ Division of Infectious Diseases, Department of Internal Medicine, Taichung Veterans General Hospital, Taichung 40705, Taiwan \\ ${ }^{5}$ Rong Hsing Research Center for Translational Medicine, National Chung Hsing University, Taichung 40227, Taiwan \\ ${ }^{6}$ Ph.D. Program in Translational Medicine, National Chung Hsing University, Taichung 40227, Taiwan
}

Correspondence should be addressed to Yao-Ting Huang; ythuang@cs.ccu.edu.tw and Po-Yu Liu; liupoyu@gmail.com

Received 21 February 2019; Revised 6 April 2019; Accepted 22 May 2019; Published 10 June 2019

Guest Editor: Alberto Antonelli

Copyright (c) 2019 Ying-Ju Chen et al. This is an open access article distributed under the Creative Commons Attribution License, which permits unrestricted use, distribution, and reproduction in any medium, provided the original work is properly cited.

Shewanella algae is an emerging pathogen widely distributed in aquatic environment. Bacteremia is a major manifestation of S. algae infections, and there are increasing reports of antibiotic-resistant strains. However, little is known about the genomic characteristics of human bacteremic $S$. algae. Here, we report the results of the whole-genome sequencing of colistin-resistant S. algae TYL, a blood isolate. Chromosome-encoded $p m r C$ associated with colistin resistance and $b l a_{\mathrm{OXA}-55}$ gene intrinsic to $S$. algae was identified. Continuous surveillance for the emergence of $S$. algae is needed.

\section{Introduction}

Shewanella algae is a nonfermenting Gram-negative bacterium and autochthonous inhabitant of aquatic environments [1]. The organism could tolerate a wide range of physiological conditions [2] and has been documented as an emerging zoonotic pathogen [3]. It has been reported to cause ulcerative disease in marine fish [4] and shellfish [5]. The clinical spectrum in human infection is broad, including bacteremia, biliary tract infection, pneumonia, and soft tissue infections [6]. Bacteremia is one of the most common presentations of $S$. algae infections, which is associated with considerable morbidity and mortality [7].

The emergence of colistin resistance has become a major public heath challenge. The major advances of sequencing technology in past decades have enabled improved understanding of the genomic background of many important pathogens and resistance determinants. High-quality genomic data have become critical for the study of pathogenesis and therapeutic intervention of infectious diseases.
Although there are increasing reports of colistinresistant $S$. algae bloodstream infections worldwide [8], little is known about the genomic characteristics of human bacteremic S. algae. Moreover, the widespread environmental nature of $S$. algae raises concern for its role as a resistance reservoir. In this study, we determined the wholegenome sequence of a colistin-resistant $S$. algae strain isolated from blood.

\section{Materials and Methods}

S. algae strain TYL was isolated on trypticase soy agar supplemented with 5\% sheep blood (Becton Dickinson, San Jose, CA, USA) from the blood of a septic patient. The strain was identified by matrix-assisted laser desorption/ionization time-of-flight mass spectrometry (MALDI-TOF MS, bioMérieux, Marcy-l'Etoile, France) and 16S ribosomal RNA gene sequencing. In brief, a PCR amplicon of the $16 \mathrm{~S}$ ribosomal RNA gene was obtained with primers $\mathrm{B} 27 \mathrm{~F}$ $\left(5^{\prime}\right.$-AGAGTTTGATCCTGGCTCAG-3 $\left.{ }^{\prime}\right)$ and U1492R (5'- 
GGTTACCTTGTTACGACTT-3') and subjected to Sanger sequencing. Sequences obtained were blasted against the bacterial 16S ribosomal RNA gene sequences in the GenBank database (http://www.ncbi.nlm.nih.gov/) using the BLASTn (optimized for MegaBLAST) algorithm. Antibacterial susceptibility testing was performed by the Vitek 2 system (bioMérieux, Marcy-l'Etoile, France) according to the manufacturer's instructions. The reference strains Escherichia coli ATCC 25922 and Pseudomonas aeruginosa ATCC 27853 were used as quality controls. The broth microdilution method was used to determine the minimum inhibitory concentration (MIC) of colistin.

Genomic DNA was extracted by using the QIAGEN Genomic-tip 100/G kit and the Genomic DNA Buffer Set (QIAGEN, Paisley, UK) then quantified by using the Qubit dsDNA HS Assay kit and the Qubit 2.0 fluorometer (Life 3 Technologies, Carlsbad, CA, USA). The indexed PCR-free library preparation was constructed using a multiplexed high-throughput sequencing TruSeq DNA Sample Preparation kit (Illumina, San Diego, CA, USA) following the standard protocol provided by the manufacturer with minor modification. Low-quality reads and bases were removed and trimmed using the Kmer-based tool DUK (http://duk. sourceforge.net/) and FASTQ Trimmer (https:/github.com/ agordon/fastx_toolkit), respectively. The reads were assembled using Velvet version 1.2.07 [9]. Wgsim 0.3.0 (https://github.com/lh3/wgsim) was then used to generate $1-3 \mathrm{~kb}$ simulated paired-end reads. The genome was assembled using the ALLPATHS-LG v. R46652 [10] and annotated using the Prokaryotic Genomes Automatic Annotation Pipeline (PGAAP) (https://www.ncbi.nlm.nih. gov/genome/annotation_prok/). Protein family analysis is performed by RPSBLAST v. 2.2.15 [11] against COGs (Clusters of Orthologous Groups of proteins) databases (Evalue cutoff 0.001) (https://warwick.ac.uk/fac/sci/moac/ people/students/peter_cock/python/rpsblast/). Homologs of the $S$. algae TYL genes were BLAST searched against the Integrated Microbial Genomes and Microbiomes database v.5.0 [12], the Virulence Factor Database [13], and the Comprehensive Antibiotic Resistance Database [14] to identify candidate virulence genes and antibiotic-resistant genes.

\section{Results and Discussion}

Whole-genome sequencing was performed using the Illumina MiSeq platform which generated 4,270,654 reads (mean read length of $301 \mathrm{bp}$ ) and a gross amount of $1,285,466,854 \mathrm{bp}$. The assembly of the draft genome sequence consists of 100 scaffolds amounting to $4,821,720 \mathrm{bp}$ (total read depth of 267-fold coverage), and the $G+C$ content is $52.95 \%$. An illustration of the genomic contents in the genome of TYL is shown in Figure 1. The maximum contig size was $234,074 \mathrm{bp}$, and the N50 size was 96,168 bp. Of the 4,304 genes predicted, 4,112 were protein-coding genes, 13 RNAs, 90 tRNAs, and 6 noncoding RNAs. The distribution of genes into COGs functional categories is presented in Table 1. This Whole Genome Shotgun project has been deposited at DDBJ/ENA/GenBank under the accession number: LVDB01000000. The version described in this paper is version LVDB01000000.

To measure the nucleotide-level genomic similarity between TYL and the other Shewanella genomes, the Average Nucleotide Identity was calculated using a modified algorithm [15]. Results from this analysis showed an Average Nucleotide Identity (ANI) value higher than 98\% with both the C6G3 (NCBI accession number: NZ_JPMA00000000.1) and MARS 14 strains (NCBI accession number: NZ_CDQH00000000.1), confirming the identification at the species level (Figure 2).

The TYL strain is resistant to colistin $(\mathrm{MIC}=32 \mu \mathrm{g} / \mathrm{ml})$ and susceptible to imipenem (MIC $\leq 0.25 \mu \mathrm{g} / \mathrm{ml}$ ), piperacillin/ tazobactam (MIC $\leq 4 \mu \mathrm{g} / \mathrm{ml})$, ceftriaxone (MIC $\leq 1 \mu \mathrm{g} / \mathrm{ml})$, ceftazidime $(\mathrm{MIC} \leq 1 \mu \mathrm{g} / \mathrm{ml})$, cefepime $(\mathrm{MIC} \leq 1 \mu \mathrm{g} / \mathrm{ml})$, gentamicin ( $\mathrm{MIC} \leq 1 \mu \mathrm{g} / \mathrm{ml})$, and amikacin $(\mathrm{MIC} \leq 2 \mu \mathrm{g} / \mathrm{ml})$.

Over the past decade, the emergence of multidrugresistant Gram-negative microorganisms increased the use of colistin as the remaining therapeutic option [17]. Nevertheless, the reports of colistin resistance globally are of great concern. The two most studied nonfermentative Gram-negative bacteria with acquired resistance to colistin are Acinetobacter baumannii and Pseudomonas aeruginosa [18]. Lipid A modification is a common mode of colistin resistance in clinical isolates belonging to these two species $[19,20]$. We identified the homolog of $p m r C$ gene associated with colistin resistance [21]. Analysis of the upstream and downstream of $p m r C$ gene was performed. We found $p m r A$ and $p m r E$ genes located downstream of $p m r C$ gene. We noted a similar arrangement in colistin-resistant S. algae MARS 14 (Figure S1). Functional genomic study on $S$. algae MARS 14 demonstrated that the colistin resistance in $S$. algae is associated with phosphoethanolamine transferase EptA-encoding pmrC gene [21]. EptA adds phosphoethanolamine to lipid A, resulting in structural modification of lipopolysaccharide and colistin resistance [21]. Studies of colistin resistance patterns of $S$. algae are limited to case series and case reports $[22,23]$. It was suggested that all $S$. algae are resistant to colistin [24]. However, there are also reports of colistin-susceptible strains [25]. Large-scale comparative genomics focus on $p m r$ genes, and colistin resistance phenotype are needed to fully exploit mechanisms of colistin resistance in $S$. algae.

The emergence of carbapenem-resistant $S$. algae is a growing concern worldwide. Carbapenemase producers are of major concern. In this study, we detected a chromosomeencoded bla $a_{\text {OXA-55 }} \beta$-lactamase gene. OXA-type carbapenemases are members of class $\mathrm{D} \beta$-lactamases with carbapenem-hydrolysing activities. The mechanisms of carbapenem resistance in $S$. algae are suggested to be caused by beta-lactamase gene $b l a_{\mathrm{OXA}-55}$ [23]. Various $b l a_{\mathrm{OXA}}$-type genes have been reported in different Shewanella species [26]. In addition, there are increasing numbers of studies on horizontal gene transfer in Shewanella that demonstrate the organism is a potential reservoir of antimicrobial resistance $[27,28]$. However, the OXA-55 $\beta$-lactamase is also present in carbapenem-susceptible $S$. algae [29]. The resistance of carbapenems in S. algae may involve a combination of OXA- 


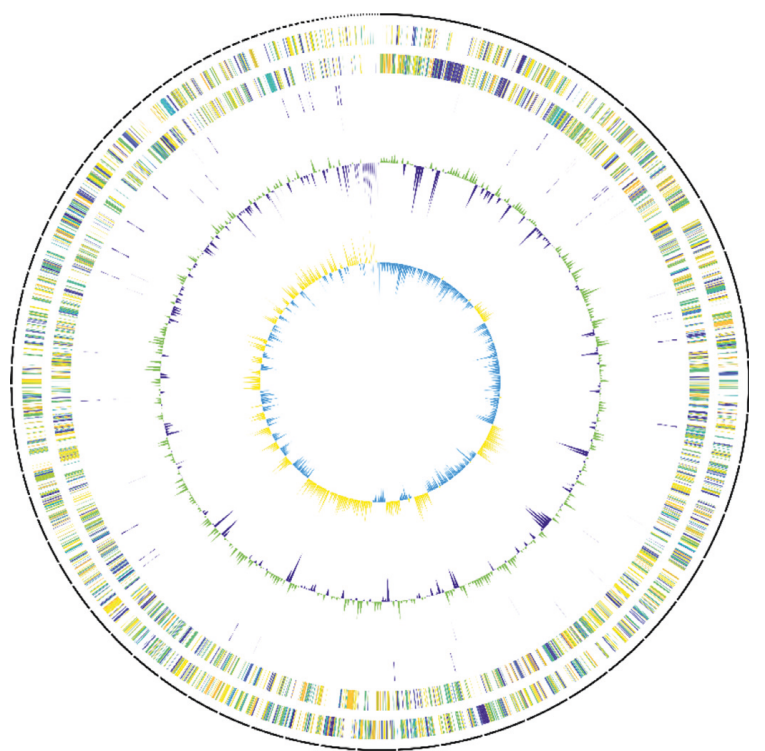

Cell cycle control, cell division, chromosome partitioning

Cell wall/membrane/envelope biogenesis

Cell motility

Posttranslational modification, protein turnover, and chaperones

Signal transduction mechanisms

Intracellular trafficking, secretion, and vesicular transport

Defense mechanisms

Extracellular structures

Nuclear structure

Cytoskeleton

RNA processing and modification

Chromatin structure and dynamics

Translation, ribosomal structure, and biogenesis

Transcription

Replication, recombination, and repair

Energy production and conversion

Amino acid transport and metabolism

Nucleotide transport and metabolism

Carbohydrate transport and metabolism

Coenzyme transport and metabolism

Lipid transport and metabolism

Inorganic ion transport and metaboism

Secondary metabolites biosynthesis, transport, and catabolism

General function prediction only

Function unknown

Multifunction

Figure 1: Circular genome map of Shewanella algae TYL. Circles from the outside to inside: (1) DNA coordinates; $(2,3)$ function-based color-coded mapping of the CDSs predicted on the forward and reverse strands. Functions are color-coded; (4) tRNA genes; (5) rRNA genes; (6) GC plot showing regions above the average (green) and below (violet); (7) GC skew showing regions above average (yellow) and below (light blue).

TABle 1: COG functional categories of Shewanella algae TYL genome.

\begin{tabular}{|c|c|c|c|}
\hline COG class & Description & Count & $\%$ \\
\hline $\mathrm{D}$ & Cell cycle control, cell division, chromosome partitioning & 37 & 0.88 \\
\hline M & Cell wall/membrane/envelope biogenesis & 223 & 5.16 \\
\hline $\mathrm{N}$ & Cell motility & 69 & 1.73 \\
\hline $\mathrm{O}$ & Posttranslational modification, protein turnover, and chaperones & 193 & 4.50 \\
\hline $\mathrm{T}$ & Signal transduction mechanisms & 223 & 5.35 \\
\hline $\mathrm{U}$ & Intracellular trafficking, secretion, and vesicular transport & 76 & 1.80 \\
\hline $\mathrm{V}$ & Defense mechanisms & 91 & 2.18 \\
\hline W & Extracellular structures & 0 & 0.00 \\
\hline A & RNA processing and modification & 2 & 0.05 \\
\hline $\mathrm{J}$ & Translation, ribosomal structure, and biogenesis & 193 & 4.64 \\
\hline K & Transcription & 248 & 5.49 \\
\hline $\mathrm{L}$ & Replication, recombination, and repair & 183 & 6.30 \\
\hline $\mathrm{C}$ & Energy production and conversion & 277 & 6.61 \\
\hline $\mathrm{E}$ & Amino acid transport and metabolism & 258 & 5.99 \\
\hline $\mathrm{F}$ & Nucleotide transport and metabolism & 89 & 2.01 \\
\hline G & Carbohydrate transport and metabolism & 109 & 2.60 \\
\hline $\mathrm{H}$ & Coenzyme transport and metabolism & 135 & 3.20 \\
\hline I & Lipid transport and metabolism & 111 & 2.51 \\
\hline $\mathrm{P}$ & Inorganic ion transport and metabolism & 257 & 5.99 \\
\hline Q & Secondary metabolites biosynthesis, transport, and catabolism & 52 & 1.23 \\
\hline $\mathrm{R}$ & General function prediction only & 0 & 0.00 \\
\hline S & Function unknown & 1,288 & 29.79 \\
\hline- & Multifunction & 80 & 1.99 \\
\hline
\end{tabular}

$55 \beta$-lactamase and another mechanism. Routine surveillance and further investigation are needed for the resistance emergence.

S. algae causes diseases in both humans and marine animals. The organism has been reported to cause various diseases in shrimp, marine fish, and shellfish $[4,5,30]$. In humans, $S$. algae infections have been associated with seafood ingestion and water exposure [6]. Severe bacteremic illness complicated with human shewanellosis is not uncommon [31]. Pathogenicity test showed the $\mathrm{LD}_{50}$ of $S$. algae was $1.8 \times 10^{4} \mathrm{CFU} / \mathrm{mL}$ for 20-day-old abalone postlarvae [4]. Hemolysis was demonstrated in the pathogenic strains of $S$. algae [30].

Hemolytic activity has long been suspected as a virulence factor of $S$. algae [32]. The S. algae TYL genome carries genes encoding hemolysin $\mathrm{A}(h l y A)$ and hemolysin 


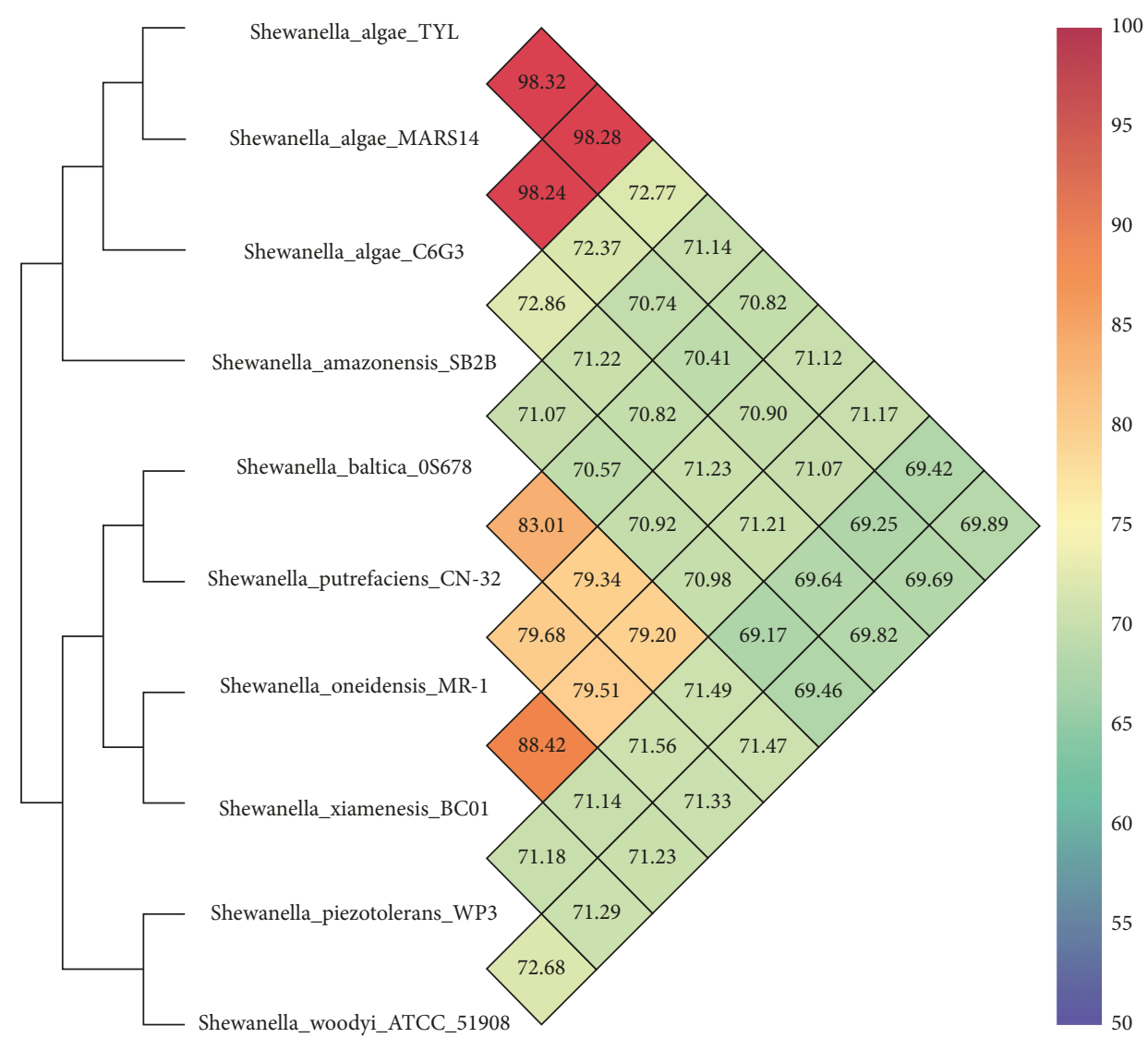

Figure 2: Heat-map and phylogenetic trees based on Average Nucleotide Identity values determined for Shewanella algae TYL and related strains. The values between two strains are given in the junction point of the diagonals departing from each strain. Figure two is reproduced from the study of Tseng et al. [16] (under the Creative Commons Attribution License/public domain).

III ( $h l y$ III). Hemolysin A belongs to RTX pore-forming toxin $\alpha$-hemolysin, which rearranges membrane permeability and causes cell lysis. Genomic analysis of $S$. algae TYL in this report supports the previous study, which demonstrated the hemolytic activity of environmental $S$. algae and thus suggested it as a possible virulence determinant [33].

Biliary tract infection is one of the major presentations and possible ports of entry in shewanellosis [34]. Bile salts possess antimicrobial activity, causing damage to lipids in the cell membrane and DNA. Hence, S. algae must be able to survive the deleterious action of bile salts. A previous study demonstrated bile resistance in S. algae [24]. In this study, we identified genes associated with bile tolerance ( $h t p B$, exbBD, and $g a l U)$. The results support the earlier genomic study, suggesting a common mechanism of bile resistance in Shewanella [16].

\section{Conclusions}

In summary, multiple resistance and virulence determinants in $S$. algae TYL were identified by wholegenome sequencing. To the best of our knowledge, this report describes the first $S$. algae blood isolate. Our data provide the impetus for further research on the zoonotic potential of S. algae.

\section{Data Availability}

This Whole Genome Shotgun project has been deposited at DDBJ/ENA/GenBank under the accession LVDB01000000. The version described in this paper is version LVDB01000000.

\section{Conflicts of Interest}

The authors declare that there are no conflicts of interest regarding the publication of this article.

\section{Acknowledgments}

We would like to thank Dr. Lee Pan for his help in Kyoto and Osaka. YTH was supported in part by a grant (106-2221-E194-056-MY3 and 107-2221-E-194 -030 -MY2) from Taiwan's Ministry of Science and Technology. YJC and PYL were supported by grants (TCVGH-PU1088101) from Taichung Veterans General Hospital.

\section{Supplementary Materials}

Figure S1: genetic context of pmrC in Shewanella algae MARS 14 and Shewanella algae TYL. The arrows indicate the positions and directions of transcription for each gene. (Supplementary Materials) 


\section{References}

[1] Z. Han, J. Sun, A. Lv et al., "Isolation, identification and characterization of Shewanella algae from reared tongue sole, Cynoglossus semilaevis Günther," Aquaculture, vol. 468, pp. 356-362, 2017.

[2] S. Y. Tseng, P. Y. Liu, Y. H. Lee et al., "The pathogenicity of Shewanella algae and ability to tolerate a wide range of temperatures and salinities," Canadian Journal of Infectious Diseases and Medical Microbiology, vol. 2018, Article ID 6976897, 9 pages, 2018.

[3] Z. Y. Wu, P. Y. Liu, S. Y. Tseng, Y. H. Lee, and S. P. Ho, "Characteristics and phylogeny of Shewanella haliotis isolated from cultivated shellfish in taiwan," Canadian Journal of Infectious Diseases and Medical Microbiology, vol. 2018, Article ID 9895148, 6 pages, 2018.

[4] C. Chen, C. Hu, X. Chen, and L. Zhang, "Identification and characterization of Shewanella algae as a novel pathogen of ulcer disease of fish Scinenops ocellata," Oceanologia Et Limnologia Sinica, vol. 34, no. 1, pp. 1-8, 2003.

[5] J. Cai, H. Chen, K. D. Thompson, and C. Li, "Isolation and identification of Shewanella alga and its pathogenic effects on post-larvae of abalone Haliotis diversicolor supertexta," Journal of Fish Diseases, vol. 29, no. 8, pp. 505-508, 2006.

[6] P.-Y. Liu, C.-F. Lin, K.-C. Tung et al., "Clinical and microbiological features of Shewanella bacteremia in patients with hepatobiliary disease," Internal Medicine, vol. 52, no. 4, pp. 431-438, 2013.

[7] A. J. Martin-Rodriguez, O. Martin-Pujol, F. Artiles-Campelo, M. Bolanos-Rivero, and U. Romling, "Shewanella spp. infections in Gran Canaria, Spain: retrospective analysis of 31 cases and a literature review," JMM Case Reports, vol. 4, no. 12, article e005131, 2017.

[8] T. Takata, H. Chikumi, S. Morishita et al., "Shewanella algae bacteremia in an end-stage renal disease patient: a case report and review of the literature," Internal Medicine, vol. 56, no. 6, pp. 729-732, 2017.

[9] D. R. Isomoto and E. Birney, "Velvet: algorithms for de novo short read assembly using de Bruijn graphs," Genome Research, vol. 18, no. 5, pp. 821-829, 2008.

[10] S. Gnerre, I. Maccallum, D. Przybylski et al., "High-quality draft assemblies of mammalian genomes from massively parallel sequence data," Proceedings of the National Academy of Sciences, vol. 108, no. 4, pp. 1513-1518, 2011.

[11] S. Altschul, W. Gish, W. Miller, E. W. Myers, and D. J. Lipman, "Basic local alignment search tool," Journal of Molecular Biology, vol. 215, no. 3, pp. 403-410, 1990.

[12] I. A. Chen, K. Chu, K. Palaniappan et al., "IMG/M v.5.0: an integrated data management and comparative analysis system for microbial genomes and microbiomes," Nucleic Acids Research, vol. 47, no. 1, pp. D666-D677, 2018.

[13] L. Chen, D. Zheng, B. Liu, J. Yang, and Q. Jin, "VFDB 2016: hierarchical and refined dataset for big data analysis-10 years on," Nucleic Acids Research, vol. 44, no. 1, pp. D694-D697, 2016.

[14] B. Jia, A. R. Raphenya, B. Alcock et al., "CARD 2017: expansion and model-centric curation of the comprehensive antibiotic resistance database," Nucleic Acids Research, vol. 45, no. 1, pp. D566-D573, 2017.

[15] I. Doshi, Y. Ouk Kim, S.-C. Park, and J. Chun, "OrthoANI: an improved algorithm and software for calculating average nucleotide identity," International Journal of Systematic and Evolutionary Microbiology, vol. 66, no. 2, pp. 1100-1103, 2016.
[16] S. Y. Tseng, K. C. Tung, J. F. Cheng et al., "Genome characterization of bile-isolated Shewanella algae ACCC," Gut Pathogens, vol. 10, no. 1, p. 38, 2018.

[17] J. Li, R. L. Nation, J. D. Turnidge et al., "Colistin: the reemerging antibiotic for multidrug-resistant gram-negative bacterial infections," The Lancet Infectious Diseases, vol. 6, no. 9, pp. 589-601, 2006.

[18] A. O. Olaitan, S. Morand, and J. M. Rolain, "Mechanisms of polymyxin resistance: acquired and intrinsic resistance in bacteria," Frontiers in Microbiology, vol. 5, p. 643, 2014.

[19] Y. Cai, D. Chai, R. Wang, B. Liang, and N. Bai, "Colistin resistance of Acinetobacter baumannii: clinical reports, mechanisms and antimicrobial strategies," Journal of Antimicrobial Chemotherapy, vol. 67, no. 7, pp. 1607-1615, 2012.

[20] N. Martis, S. Leroy, and V. Blanc, "Colistin in multi-drug resistant Pseudomonas aeruginosa blood-stream infections," Journal of Infection, vol. 69, no. 1, pp. 1-12, 2014.

[21] A. A. Telke and J.-M. Rolain, "Functional genomics to discover antibiotic resistance genes: the paradigm of resistance to colistin mediated by ethanolamine phosphotransferase in Shewanella algae MARS 14," International Journal of Antimicrobial Agents, vol. 46, no. 6, pp. 648-652, 2015.

[22] J. M. Janda and S. L. Abbott, "The genus Shewanella: from the briny depths below to human pathogen," Critical Reviews in Microbiology, vol. 40, no. 4, pp. 293-312, 2014.

[23] K. Yousfi, S. Bekal, V. Usongo, and A. Touati, "Current trends of human infections and antibiotic resistance of the genus Shewanella," European Journal of Clinical Microbiology \& Infectious Diseases, vol. 36, no. 8, pp. 1353-1362, 2017.

[24] H. M. Holt, B. Gahrn-Hansen, and B. Bruun, "Shewanella algae and Shewanella putrefaciens: clinical and microbiological characteristics," Clinical Microbiology and Infection, vol. 11, no. 5, pp. 347-352, 2005.

[25] M. Gressier, D. Mbayo, H. Deramond, F. Grados, F. Eb, and B. Canarelli, "First case of human spondylodiscitis due to Shewanella algae," International Journal of Infectious Diseases, vol. 14, no. 3, pp. e261-e264, 2010.

[26] A. Antonelli, D. M. Di Palo, A. Galano et al., "Intestinal carriage of Shewanella xiamenensis simulating carriage of OXA-48-producing Enterobacteriaceae," Diagnostic Microbiology and Infectious Disease, vol. 82, no. 1, pp. 1-3, 2015.

[27] Y. Fang, Y. Wang, Z. Li et al., "Distribution and genetic characteristics of SXT/R391 integrative conjugative elements in Shewanella spp. from China," Frontiers in Microbiology, vol. 9, p. 920, 2018.

[28] A. B. Jousset, L. Dabos, R. A. Bonnin et al., "CTX-M-15Producing Shewanella species clinical isolate expressing OXA-535, a chromosome-encoded OXA-48 variant, putative progenitor of the plasmid-encoded OXA-436," Antimicrobial Agents and Chemotherapy, vol. 62, no. 1, 2018.

[29] J. Walther-Rasmussen and N. Høiby, "OXA-type carbapenemases," Journal of Antimicrobial Chemotherapy, vol. 57, no. 3, pp. 373-383, 2006.

[30] L. Shufang, J. Zhang, Q. Dequan, Y. Shiping, and Z. Huang, "Biological characteristics and pathogenicities of Shewanella algae and Shewanella abalone from babylonia," Agricultural Science \& Technology, vol. 16, no. 9, pp. 1845-1859, 2015.

[31] F. Zhang, Y. Fang, F. Pang et al., "Rare Shewanella spp. associated with pulmonary and bloodstream infections of cancer patients, China: a case report," BMC Infectious Diseases, vol. 18, no. 1, p. 454, 2018.

[32] G. P. Richards, M. A. Watson, E. J. Crane 3rd, I. G. Burt, and D. Bushek, "Shewanella and photobacterium spp. in oysters 
and seawater from the Delaware Bay," Applied and Environmental Microbiology, vol. 74, no. 11, pp. 3323-3327, 2008.

[33] Z. Y. Wu, S. P. Ho, J. F. Cheng et al., "Whole-genome characterization of Shewanella algae strain SYT3 isolated from seawater reveals insight into hemolysis," Future Microbiology, vol. 13, no. 16, pp. 1709-1717, 2018.

[34] J.-H. Byun, H. Park, and S. Kim, "The phantom menace for patients with hepatobiliary diseases: Shewanella haliotis, often misidentified as Shewanella algae in biochemical tests and MALDI-TOF analysis," Japanese Journal of Infectious Diseases, vol. 70, no. 2, pp. 177-180, 2017. 


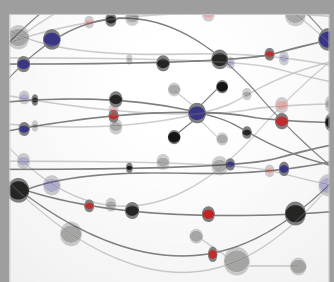

The Scientific World Journal
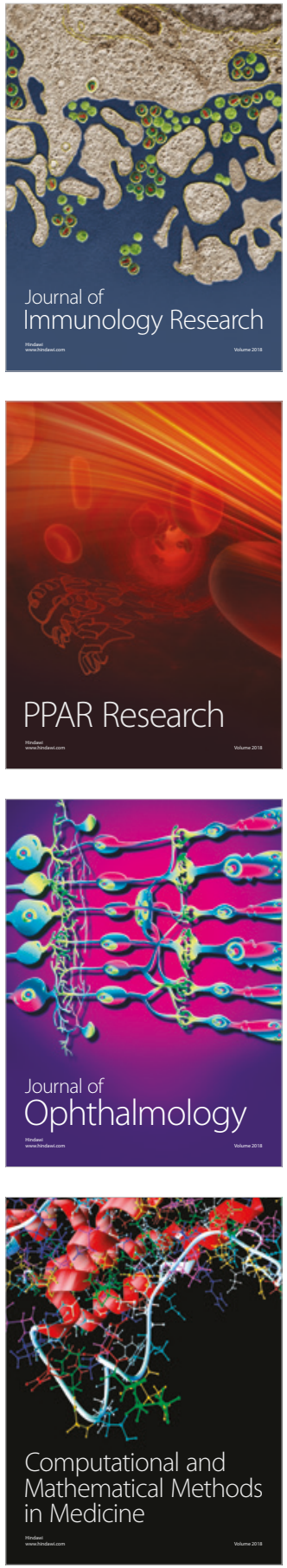

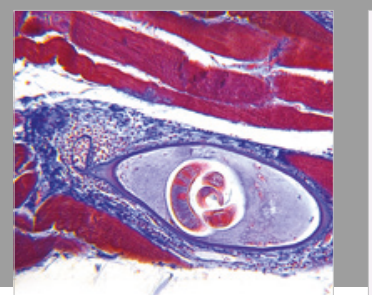

Gastroenterology Research and Practice

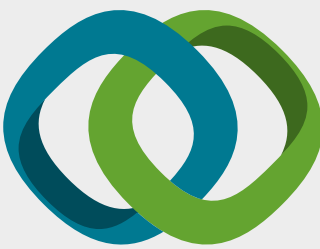

\section{Hindawi}

Submit your manuscripts at

www.hindawi.com
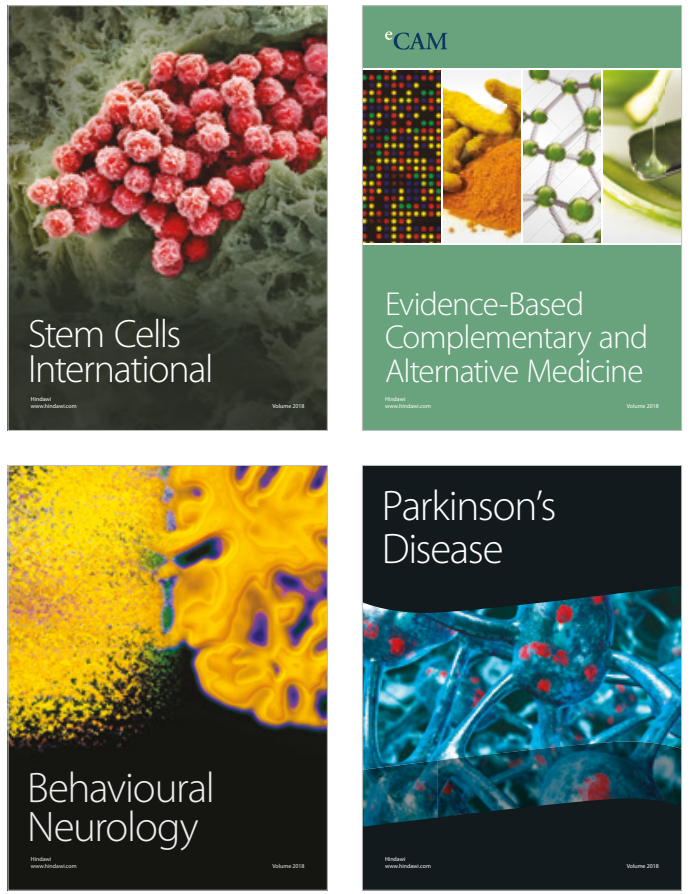

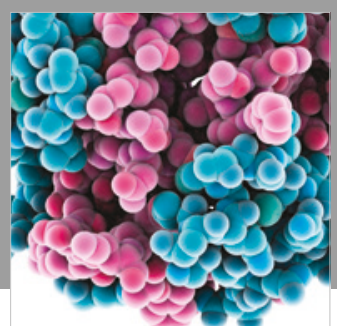

ournal of

Diabetes Research

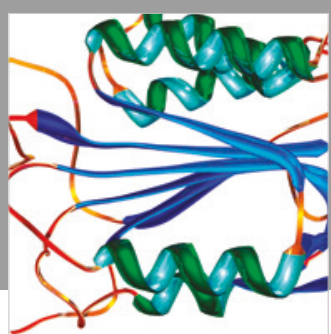

Disease Markers
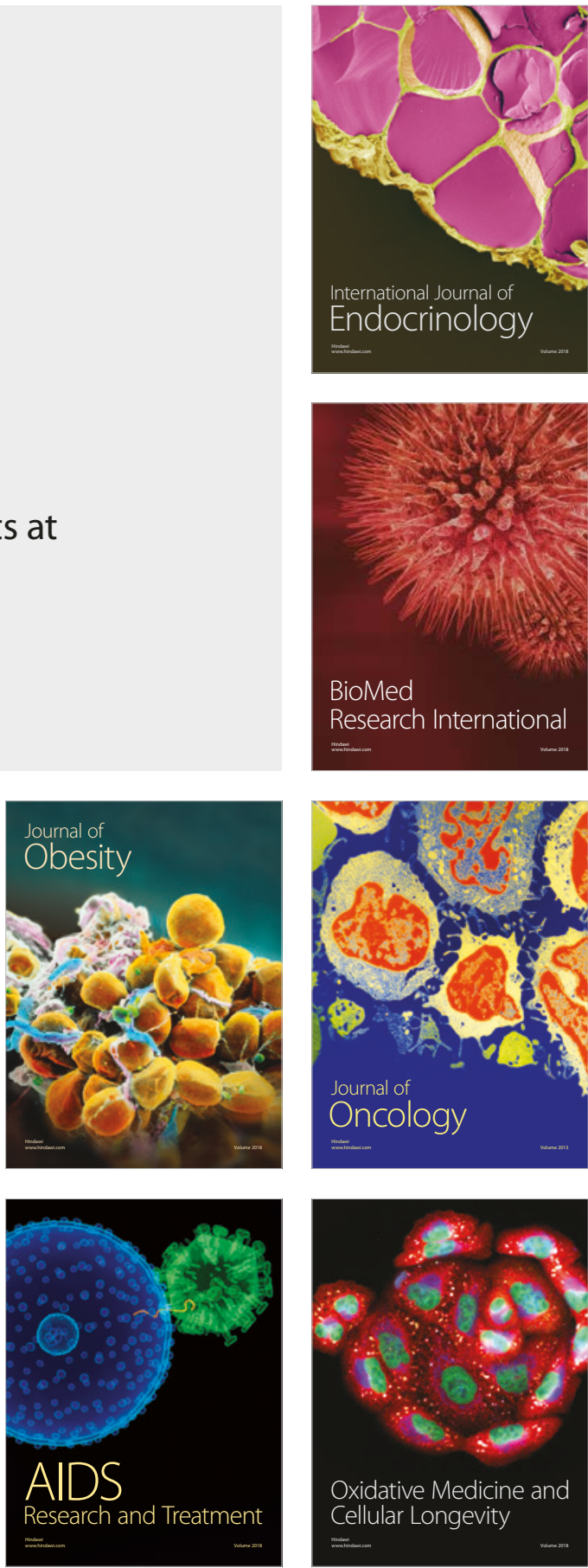